Note

\title{
The Preparation of Ag Nanoparticle-Modified Single-Walled Carbon Nanotubes and Their Antibacterial Activity
}

\author{
TOMOMI KOSAKA ${ }^{1 *}$, AKIE OHGAMI ${ }^{1}$, TAKAKO NAKAMURA ${ }^{2}$, \\ TSUGUYORI OHANA ${ }^{2}$ AND MASATOU ISHIHARA ${ }^{2}$
}

\author{
'Department of Chemistry, Tokyo Gakugei University, 4-1-1, Nukuikita-machi, Koganei, \\ Tokyo 184-8501, Japan \\ ${ }^{2}$ Research Center for Advanced Carbon Materials, National Institute of Advanced Industrial Science \\ and Technology (AIST), 1-1-1, Higashi, Tsukuba, Ibaraki 305-8565, Japan
}

Received 10 September, 2008/Accepted 25 April, 2009

\begin{abstract}
The sidewall modified single-walled carbon nanotubes (SWNTs) with amino-containing substituents were prepared using the radicals generated by the photolysis of acetonitrile. A subsequent treatment of modified SWNTs with the Ag colloid gave an attachment of $\mathrm{Ag}$ nanoparticles on the surface of SWNTs through the functionalized linkages. The Ag nanoparticle-modified SWNTs evaluated by antibacterial tests showed strong activities against Escherichia coli and Staphylococcus aureus. However, the use of Ag nanoparticlemodified SWNTs on simulated body fluid exhibited weaker antibacterial activity.
\end{abstract}

Key words : Single-walled carbon nanotubes/Chemical modification/Antibacterial activity/ Ag nanoparticles.

Single-walled carbon nanotubes (SWNTs) have a wide range of applications due to their unique electronic, optical, mechanical (Dresselhaus et al., 1996; Saito et al., 1998), antimicrobial (Kang et al., 2007) and metallic or semiconducting properties depending on their diameter and helicity. The chemical functionalization of the sidewall of SWNTs has been expected to add novel functions to the original behavior.

Thiol-, thio- and amino-containing materials have attracted much attention because of their properties derived from the presence of sulfur- and nitrogencontaining functional groups. It is well-known that organic compounds containing thio- and thiol groups are important materials for biologically active substances and show good affinities to gold to give a self-assembled monolayer. Amino functionalities play an important role in constructing complex structures

${ }^{*}$ Corresponding author. Tel : +81-42-329-7511, Fax : +8142-329-7511. with a combination of other functional groups. The sidewall modification of carbon nanotube surfaces with amino substituents has been investigated by the use of ammonia with the ball-milling method (Lin et al., 2003), a substitution of fluorinated SWNTs with diamines (Stevens et al., 2003), a conversion of nitro moieties to aniline (Guo and Li, 2005) and a further functionalization process of carboxylated SWNTs (Ramanathan et al., 2005; Gromov et al., 2005). However, these methods would create problems about the handling of the reactions due to the use of hazardous reactants and the necessity of multi-step reactions. Previously, we reported on the photochemical modification of SWNTs with fluorine functionalities by the use of radical reactions, which took place under mild conditions and gave no heavy modification (Nakamura et al., 2004) compared with conventional methods described above.

It is known that acetonitrile is photolysed to give $\mathrm{CH}_{2} \mathrm{CN}$ radicals upon irradiation with VUV light (Lesiecki and Guillory, 1978 ; Halpern and Tang, 
1985 ; Cheng et al., 2000). Carbon nanotubes (CNTs) have poor solubility and tend to aggregate in the water. Then we considered that surface modification of CNTs has been effective to their antibacterial activity. Akasaka et al. (2005) reported that the apatite coating on multi-walled carbon nanotubes is achieved using simulated body fluid (SBF). SBF has been frequently used for the apatite coating to increase bioactivity and has been successfully applied to implant materials such as special dental and medical devices. Nonami et al. (2003) prepared apatitecoated $\mathrm{TiO}_{2}$ composite and reported that the composite has better function of absorbing and decomposing acetaldehyde, nitrogen oxide and Escherichia coli than the uncoated $\mathrm{TiO}_{2}$. Accordingly, we considered that an apatite coating on Ag modified SWNTs might improve their antibacterial activity. In this paper, we prepared novel Ag modified SWNTs with amino substituents via the radical reactions produced by using the photolysis of acetonitrile and the attachment of Ag nanoparticles. We also investigated the antibacterial activity of the Ag modified SWNTs and those treated with SBF.

SWNTs (0.9-1.2 nm diameter, 10-50 $\mu \mathrm{m}$ length) and $\mathrm{NaBH}_{4}$ were purchased from Aldrich Co. Inc. Acetonitrile and $10 \mathrm{mM}$ aqueous solution of $\mathrm{AgNO}_{3}$ were obtained from Kanto Chemical Co. Inc. A suspension of purified SWNTs (hereafter referred as pSWNTs $)$ in acetonitrile $(4 \mathrm{ml})$ was irradiated with a Xe excimer lamp (USHIO UER2OH-172C) for $3 \mathrm{~h}$ at room temperature under an argon atmosphere with stirring. After removing the acetonitrile solution from the reaction mixture, the residual powders were washed with acetonitrile. The attachment of silver nanoparticles on the surface of amino-functionalized SWNTs (hereafter referred as amino-SWNTs) was carried out by treating the amino-SWNTs $(1.7 \mathrm{mg}$ ) with $10 \mathrm{mM}$ aqueous solution of $\mathrm{AgNO}_{3}(5 \mathrm{ml})$, then with $40 \mathrm{mM}$ aqueous solution of $\mathrm{NaBH}_{4}(10 \mathrm{ml})$ with stirring for $5 \mathrm{~min}$. After finishing the reaction, the aqueous solution was removed from the reaction mixture, and then the samples were washed with water.

The Ag nanoparticle-modified SWNTs (hereafter referred as Ag-SWNTs) were dispersed in Dulbecco's phosphate-buffered saline (D-PBS) at a concentration of $10 \mathrm{mg} / \mathrm{l}$ by ultrasonication for 10 min. Then, the apatite crystallites were grown by immersing the samples at $36^{\circ} \mathrm{C}$ for $2 \mathrm{~h}$. The D-PBS powder was purchased from Invitrogen Japan K.K. The composition of D-PBS was $\mathrm{NaCl}(8000 \mathrm{mg} / \mathrm{l}), \mathrm{CaCl}_{2}$ $(100 \mathrm{mg} / \mathrm{l}), \mathrm{KH}_{2} \mathrm{PO}_{4}(200 \mathrm{mg} / \mathrm{l}), \mathrm{Na}_{2} \mathrm{HPO}_{4}$ (1150 $\mathrm{mg} / \mathrm{l}), \mathrm{KCl}(200 \mathrm{mg} / \mathrm{l}), \mathrm{MgCl}_{2}(47 \mathrm{mg} / \mathrm{l})$. The apatitecoated Ag-SWNTs were referred as Ag-HA-SWNTs.

The obtained samples were analyzed by diffusion reflectance infra-red Fourier transform spectroscopy (DRIFT), X-ray photoelectron spectroscopy (XPS), scanning electron microscopy (SEM), Raman spectroscopy, mass and UV-vis-NIR spectrometers.

DRIFT spectra were recorded with JASCO FT/IR680 Plus spectrometers. XPS was measured by a PHI ESCA model5800 using aluminum $\mathrm{K} \alpha$ radiation. The chemically modified samples were analyzed using the mass spectrometer (JEOL JMS-AMSUN200) with the direct insertion method. Raman spectra were measured with JASCO Laser Raman Spectrometers NRS2100 using the $514.5 \mathrm{~nm}$ line of an argon ion laser with a power of $100 \mathrm{~mW}$. SEM was used to examine the surface morphology before and after the reactions. Samples were mounted on an Al stub using conducting carbon paste before examination in a Hitachi S-5000 model. UV-vis-NIR spectra were measured by Parkin-Elmer Lambda 900.

Escherichia coli NBRC 3972 and Staphylococcus aureus subsp. aureus NBRC 12732 were used for antibacterial tests. One loopful of the refrigerated bacteria was cultured in $1 \mathrm{ml}$ nutrient broth at $36^{\circ} \mathrm{C}$ for 24 h. After being cultured, the bacteria were suspended in sterile deionized water to a concentration from $10^{6}$ to $10^{7}$ cells $/ \mathrm{ml}$. Suspensions of p-SWNTs (1.1 $\mathrm{mg}$ ), Ag-SWNTs (1.1 mg) and Ag-HA-SWNTs $(1.0 \mathrm{mg})$ with sterile deionized water $(900 \mu \mathrm{l})$, a suspension of bacteria $(100 \mu \mathrm{I})$ and nutrient broth (2 $\mu \mathrm{l})$ were allowed to stand in the incubator at $36^{\circ} \mathrm{C}$ using $1 \mathrm{ml}$ micro tubes. Then, the initial concentration of bacteria was from $10^{5}$ to $10^{6} \mathrm{cells} / \mathrm{ml}$. The contaminated water was diluted to $10^{2} \sim 10^{4}$ cells $/ \mathrm{ml}$ by sterile deionized water using $1 \mathrm{ml}$ micro tubes at each sampling. After $50 \mu \mathrm{l}$ of the contaminated water in a micro tube had been dropped and spread on a growth medium-standard method agar in a petri dish, the bacteria formed small visible colonies after $24 \mathrm{~h}$ incubation at $36^{\circ} \mathrm{C}$. The number of bacteria colonies was counted.

XPS spectra of SWNTs were measured before and after irradiation in the presence of acetonitrile (Fig. 1), showing a new peak at $400.5 \mathrm{eV}$ of nitrogen 1s together with the $\mathrm{sp}^{2}$ carbon peak at $284.6 \mathrm{eV}$ after irradiation. In the carbon $1 \mathrm{~s}$ region, the carbon peak was deconvoluted to the mixture of $\mathrm{sp}^{2}$ carbon and carbon atoms bound to nitrogen atoms with a higher binding energy, compared with the spectra of p-SWNTs.

IR spectra of the samples before and after the treatment of irradiation with acetonitrile are shown in Fig. 2. New peaks at 2922 and $2856 \mathrm{~cm}^{-1}$ with a $\mathrm{C}-\mathrm{H}$ stretching band and at $3434 \mathrm{~cm}^{-1}$ with a $-\mathrm{NH}_{2}$ stretching band were observed, accompanied with a $\mathrm{C}=\mathrm{C}$ bond peak $\left(1631 \mathrm{~cm}^{-1}\right)$, as contrasted with no peaks in the case of $\mathrm{p}$-SWNTs. 


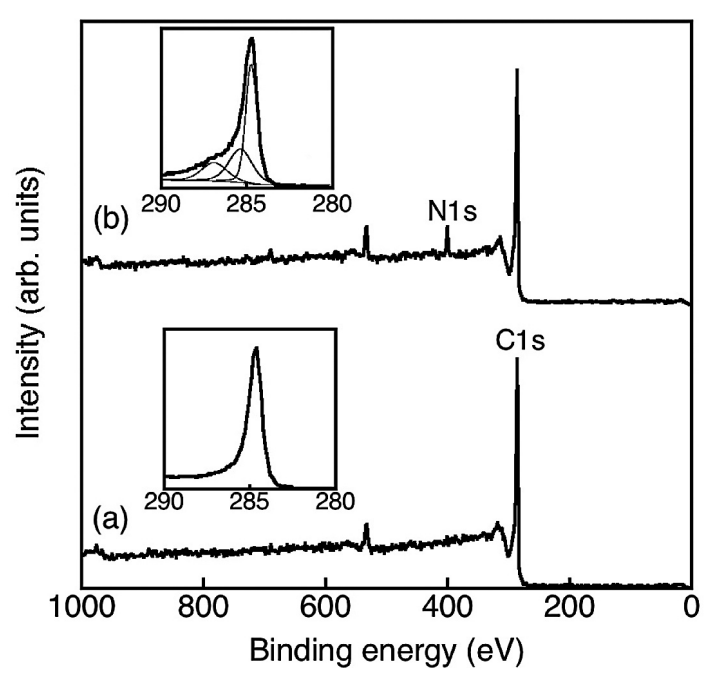

FIG. 1. XPS spectra of SWNTs were measured (a) before and (b) after irradiation in the presence of acetonitrile. C1s and $\mathrm{N} 1 \mathrm{~s}$ denote the carbon $1 \mathrm{~s}$ and nitrogen $1 \mathrm{~s}$ orbital photoelectron peaks, respectively. The inset graphs show the carbon 1s region.

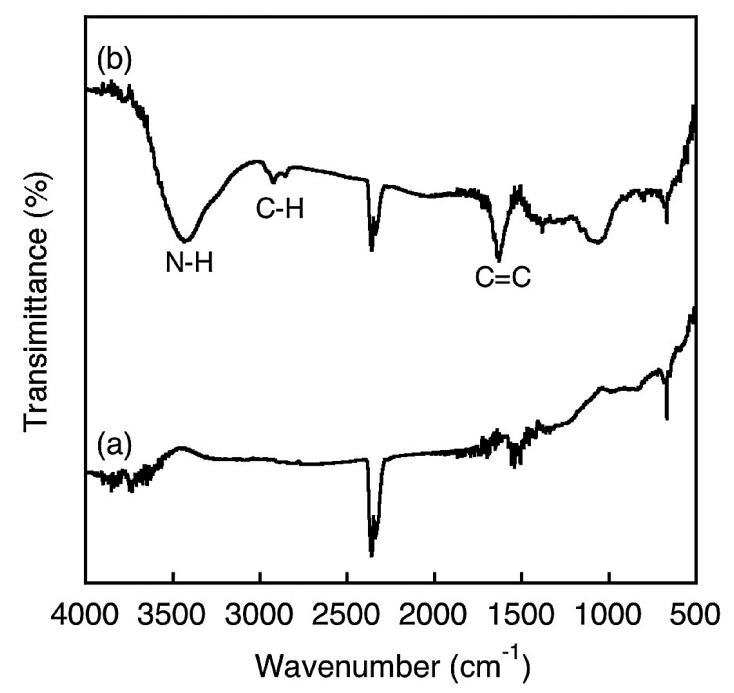

FIG. 2. IR spectra of (a) p-SWNTs and (b) amino-SWNTs.

The mass spectrum of components generated by heating amino-SWNTs to $300^{\circ} \mathrm{C}$ is shown in Fig.3a. A parent peak at $\mathrm{m} / \mathrm{z} 43\left(\mathrm{C}_{2} \mathrm{H}_{5} \mathrm{~N}^{+}\right)$was ascribed to the $\mathrm{CH}_{2} \mathrm{CH}_{2} \mathrm{NH}$ group with fragmentation peaks $\mathrm{m} / \mathrm{z} 29$ $\left(\mathrm{C}_{2} \mathrm{H}_{5}^{+}\right)$consistent with the presence of the $-\mathrm{CH}_{2} \mathrm{CH}_{2} \mathrm{NH}_{2}$ moiety. Moreover, the covalent functionalization of SWNTS was confirmed by UV-visNIR measurements in DMF as shown in Fig. 3b. It was known that a covalent sidewall modification on the surface of CNTs would result in changes in their original electronic behavior. In comparing the spectra of p-SWNTs and amino-SWNTs, it was found that the
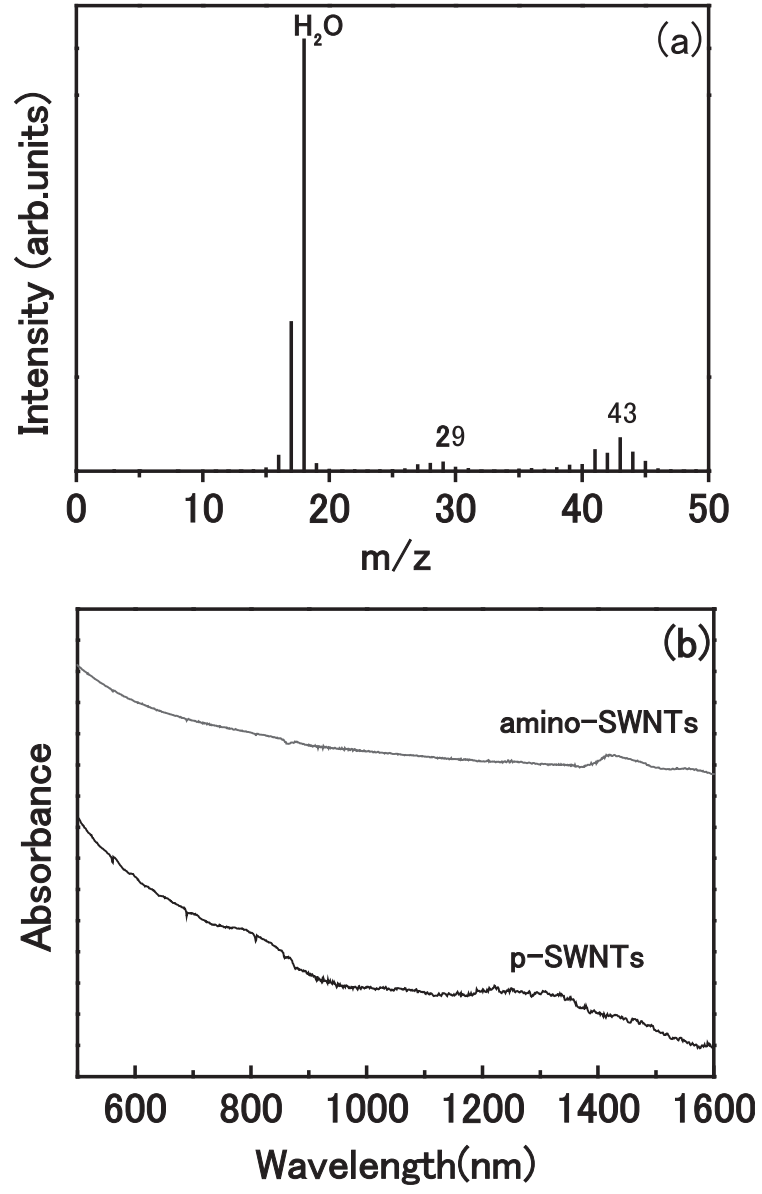

FIG. 3. (a) Mass spectrum of amino-SWNTs after heating to $300^{\circ} \mathrm{C}$. (b) UV-vis-NIR spectra of p-SWNTs and aminoSWNTS.

sidewall functionalization of SWNTs with amino substituents partly suppressed the original absorption of SWNTs (Park et al., 2003).

Figure 4 shows the Raman spectra of p-SWNTs and amino-SWNTs. Characteristic peaks were observed at 180, 165 and $149 \mathrm{~cm}^{-1}$ due to a breathing mode of p-SWNTs, accompanied with typical peaks at 1574 and $1337 \mathrm{~cm}^{-1}$ attributed to $G$ and $D$ band, respectively. The vibration mode at $G$ band was attributed to the characteristic phonon dispersion of graphite and the one at $D$ band arose from the disorder in the structure in the plane with graphite. In the case of amino-SWNTs, the breathing mode was suppressed by introduction of amino substituents. It was found that the peak intensity of the D band in aminoSWNTs increased in comparison to that of the pSWNTs by covalent functionalization.

These analytical results suggest a plausible reaction mechanism as shown in Scheme 1. Hydrogen and $\mathrm{CH}_{2} \mathrm{CN}$ radicals, generated by the photolysis of acetonitrile, reacted with the $\mathrm{C}=\mathrm{C}$ bond on SWNTs 


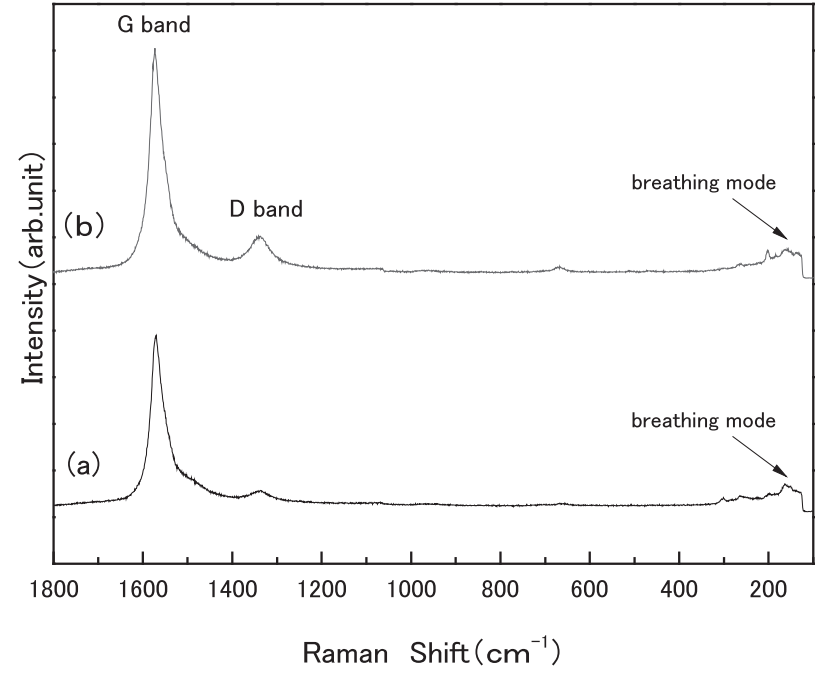

FIG. 4. Raman spectra of (a) p-SWNTs and (b) aminoSWNTS.

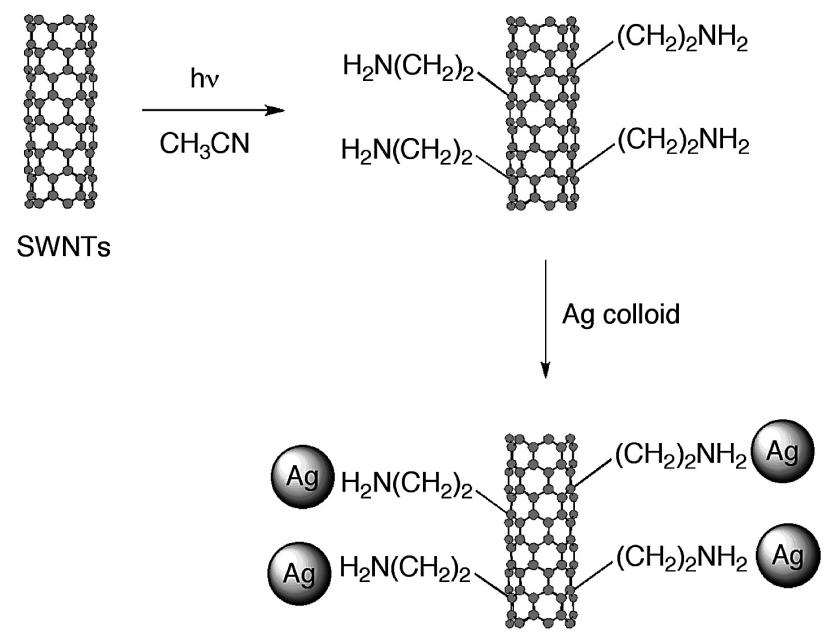

Scheme. 1. Chemical modification of SWNTs by photolysis of acetonitrile and subsequent treatment with $\mathrm{Ag}$ nanoparticles.

sidewall, then hydrogen radicals reacted with the $\mathrm{CH}_{2} \mathrm{CN}$ moiety to give aminated SWNTs. This method is useful for the introduction of amino functionalities on the sidewall of SWNTs.

The attachment of $\mathrm{Ag}$ nanoparticles to aminoSWNTs formed by the photoreaction in acetonitrile was performed by the treatment with Ag colloids. As shown in Fig. $5 \mathrm{a}$, the XPS spectrum shows a new peak at $369.0 \mathrm{eV}$ of silver $3 \mathrm{~d}$ together with the $\mathrm{sp}^{2}$ carbon peak at $284.6 \mathrm{eV}$ and nitrogen peak at 400.5 $\mathrm{eV}$. The atomic ratio of $\mathrm{C}, \mathrm{N}$ and $\mathrm{Ag}$ was estimated to be 93:2:5 in the Ag-SWNTs. As shown in Figure 5b and $\mathrm{c}$, a SEM image of amino-SWNTs with Ag nanoparticles through the amino linkage indicates a

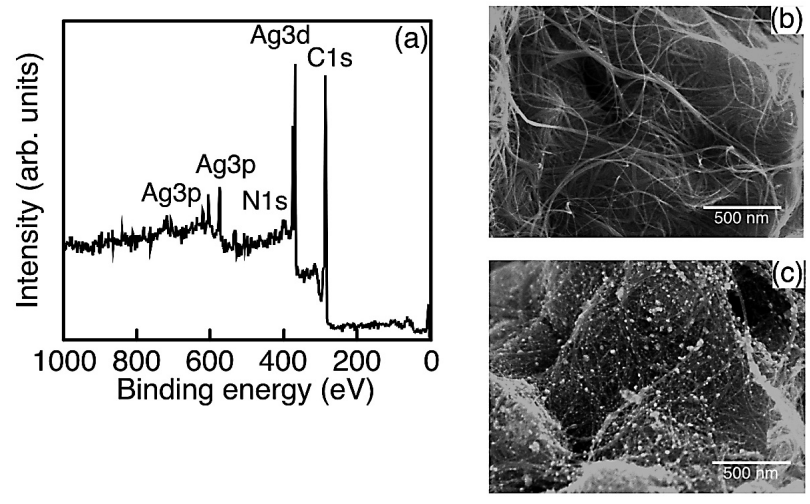

FIG. 5. (a) XPS spectrum of Ag-SWNTs. SEM images of (b) p-SWNTs and (c) Ag-SWNTs.

Ag3p, Ag3d denote silver $3 p$ and $3 d$ orbital photoelectron peaks, respectively.

high dispersion of $\mathrm{Ag}$ nanoparticles on sidewall SWNTs and reveals no significant changes after the introduction of the substituents compared with that of p-SWNTs.

Antibacterial activity tests of p-SWNTs and AgSWNTs were performed against $E$. coli and $S$. aureus as shown in Table 1 and Table 2, respectively. In the case of the absence of SWNTs, the number of bacteria did not increase after $3 \mathrm{~h}$. On the other hand, AgSWNTs showed a strong bactericidal activity, in which colonies of $E$. coli and $S$. aureus were not observed in $2 \mathrm{~h}$ and $3 \mathrm{~h}$, respectively. These results indicate that Ag-SWNTs are useful as an antibacterial agent owing to the presence of $\mathrm{Ag}$ nanoparticles known to play an important role in strong bactericidal activities as reported previously (Matsumura et al., 2003 ; Sondi and Salopek-Sondi, 2004). In the case of Ag-HA-SWNTs, it was found that the SBF treatment resulted in a weaker antibacterial activity of AgSWNTs. According to XPS analysis as shown in Figure 6 , it is confirmed that hydroxyapatite $(H A)$ is formed on the surface of samples by soaking in SBF for $2 \mathrm{~h}$ at $36^{\circ} \mathrm{C}$. However, we could not detect on improvement in the antibacterial activity contrary to our expectations. We also detected that colonies of $S$. aureus were not observed after $24 \mathrm{~h}$ if $\mathrm{p}$-SWNTs were in the suspension. Kang et al. (2007) reported that highly purified SWNTs have strong antimicrobial activity against $E$. coli $\mathrm{K} 12$. Our results against $S$. aureus were consistent with their conclusion. However, it is considered that further detailed experiments that include the interaction between p-SWNTs, Ag-SWNTs, Ag-HA- SWNTs and bacteria are needed.

The photolysis of acetonitrile in the presence of SWNTs led to a sidewall modification of the surface to introduce amino functional groups, respectively, 
TABLE 1. The antibacterial activities of p-SWNTs, Ag-SWNTs and Ag-HA-SWNTs against E.coli. Values are shown as mean \pm standard deviation obtained from three sets of data.

\begin{tabular}{lccccc}
\multicolumn{7}{c}{ Oh } & 1h & $2 \mathrm{~h}$ & $3 \mathrm{~h}$ & (cfu/ml) \\
\hline & $2.0 \mathrm{E}+06$ & $2.0 \mathrm{E}+06$ & $2.0 \mathrm{E}+06$ & $3.7 \mathrm{E}+06$ & $1.1 \mathrm{E}+06$ \\
Control & $\pm 2.0 \mathrm{E}+05$ & $\pm 1.2 \mathrm{E}+05$ & $\pm 1.7 \mathrm{E}+05$ & $\pm 4.7 \mathrm{E}+05$ & $\pm 1.4 \mathrm{E}+05$ \\
& $2.0 \mathrm{E}+06$ & $1.8 \mathrm{E}+06$ & $1.7 \mathrm{E}+06$ & $2.6 \mathrm{E}+05$ & $1.4 \mathrm{E}+06$ \\
p-SWNTs & $\pm 2.0 \mathrm{E}+05$ & $\pm 2.8 \mathrm{E}+05$ & $\pm 1.9 \mathrm{E}+05$ & $\pm 0.0 \mathrm{E}+00$ & $\pm 5.5 \mathrm{E}+05$ \\
& $7.9 \mathrm{E}+05$ & $8.0 \mathrm{E}+02$ & & & $<200$ \\
Ag-SWNTs & $\pm 1.4 \mathrm{E}+04$ & $\pm 5.7 \mathrm{E}+02$ & $<200$ & $<200$ & \\
& $9.8 \mathrm{E}+05$ & $8.3 \mathrm{E}+04$ & $2.4 \mathrm{E}+02$ & & $<200$ \\
Ag-HA-SWNTs & $\pm 5.7 \mathrm{E}+04$ & $\pm 5.7 \mathrm{E}+03$ & $\pm 0.0 \mathrm{E}+00$ & $<200$ & \\
& & &
\end{tabular}

TABLE 2. The antibacterial activities of p-SWNTs, Ag-SWNTs and Ag-HA-SWNTs against S.aureus. Values are shown as mean \pm standard deviation obtained from three sets of data.

$(\mathrm{cfu} / \mathrm{ml})$

\begin{tabular}{lccccc}
\hline & Oh & $1 \mathrm{~h}$ & $2 \mathrm{~h}$ & $3 \mathrm{~h}$ & $24 \mathrm{~h}$ \\
\hline Control & $1.8 \mathrm{E}+06$ & $1.9 \mathrm{E}+06$ & $1.7 \mathrm{E}+06$ & $1.7 \mathrm{E}+06$ & $7.7 \mathrm{E}+06$ \\
& $\pm 1.3 \mathrm{E}+05$ & $\pm 3.2 \mathrm{E}+05$ & $\pm 1.4 \mathrm{E}+05$ & $\pm 9.0 \mathrm{E}+04$ & $\pm 1.8 \mathrm{E}+06$ \\
p-SWNTs & $1.8 \mathrm{E}+06$ & $8.1 \mathrm{E}+05$ & $2.1 \mathrm{E}+05$ & $5.0 \mathrm{E}+04$ & $<200$ \\
& $\pm 1.3 \mathrm{E}+05$ & $\pm 4.2 \mathrm{E}+04$ & $\pm 7.6 \mathrm{E}+04$ & $\pm 1.4 \mathrm{E}+04$ & \\
Ag-SWNTs & $1.9 \mathrm{E}+06$ & $8.7 \mathrm{E}+04$ & $1.6 \mathrm{E}+03$ & $<200$ & $<200$ \\
& $\pm 1.8 \mathrm{E}+05$ & $\pm 9.9 \mathrm{E}+03$ & $\pm 0.0 \mathrm{E}+00$ & & \\
Ag-HA-SWNTs & $9.9 \mathrm{E}+05$ & $7.4 \mathrm{E}+05$ & $3.4 \mathrm{E}+05$ & $1.1 \mathrm{E}+05$ & $<200$ \\
& $\pm 1.8 \mathrm{E}+05$ & $\pm 1.9 \mathrm{E}+05$ & $\pm 3.4 \mathrm{E}+04$ & $\pm 2.0 \mathrm{E}+04$ & \\
\hline
\end{tabular}

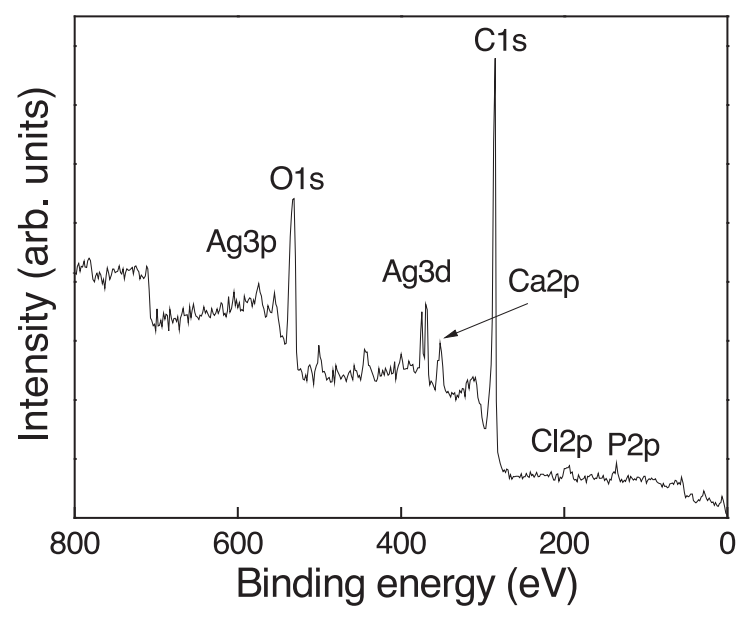

FIG. 6. XPS spectra of Ag-SWNTs after SBF treatment. $\mathrm{O} 1 \mathrm{~s}, \mathrm{Ca} 2 \mathrm{p}, \mathrm{Cl} 2 \mathrm{p}$ and P2p denote the oxygen 1s, calcium $2 p$, chlorine $2 p$ and phosphorus $2 p$ orbital photoelectron peaks, respectively.

via a one-step reaction. These methods are useful for the modification of sidewall SWNTs with organic functional groups because byproducts are not formed and SWNTs electronic behavior is not destroyed unlike in the case of heavy modification. The modified
SWNTs with amino functionalities could be modified further by the attachment of $\mathrm{Ag}$ nanoparticles on sidewall SWNTs. The Ag nanoparticle-functionalised SWNTs evaluated by antibacterial tests with E.coli and S.aureus showed strong antibacterial activities. The apatite-coated Ag-SWNTs exhibited weaker antibacterial activity. However, it is considered that further detailed experiments to investigate the effect of the apatite coating on SWNTs on the absorbing function of the bacteria are needed.

\section{REFERENCES}

Akasaka, T., and Watari, F. (2005) Nano-architecture on Carbon Nanotube Surface by Biomimetic Coating. Chemistry Letters, 34, 826-827.

Chuang, C.C., Wu, W.C., Lee, M.X., and Lin, J.L. (2000) Adsorption and photochemistry of $\mathrm{CH}_{3} \mathrm{CN}$ and $\mathrm{CH}_{3} \mathrm{CONH}_{2}$ on powdered $\mathrm{TiO}_{2}$. Phys. Chem. Chem. Phys., 2, 3877-3882.

Dresselhaus, M.S., Dresselhaus, G., and Eklund, P.C. (1996) Science of Fullerene and Carbon Nanotubes. Academic Press, San Diego.

Gromov, A., Dittmer, S., Svensson, J., Nerushev, O.A., PerezGarcia, S.A., LiceaJimenez, L., Rychwalski, R., and Campbell, E.E.B. (2005) Covalent aminofunctionalisation of single-wall carbon nanotubes. J. 
Mater. Chem., 15, 3334-3339.

Guo, D.J., and Li, H.L. (2005) Highly dispersed Ag nanoparticles on functional MWNT surfaces for methanol oxidation in alkaline solution. Carbon, 43, 1259-1264.

Halpern, J.B., and Tang, X. (1985) Production of CN $\left(A^{2} \Pi_{i}\right)$ in the photolysis of acetonitrile at $158 \mathrm{~nm}$. Chem. Phys. Lett., 122, 294-299.

Kang, S., Pinault, M., Pfefferle, L.D., and Elimelech, M. (2007) Single-Walled Carbon Nanotubes Exhibit Strong Antimicrobial Activity. Langmuir, 23, 8670-8673.

Lesiecki, M.L., and Guillory, W.A. (1978) Low pressure infrared multiple-photon photochemistry of acetonitrile. J. Chem. Phys., 69, 4572-4587.

Lin, S., Bajpai, V., Ji, T., and Dai, L.M. (2003) Chemistry of Carbon Nanotube. Aust. J. Chem., 56, 635-651.

Matsumura, Y., Yoshitaka, Y., Kunisaki, S., and Tsuchido, T. (2003) Mode of Bactericidal Action of Silver Zeolite and Its Comparison with That of Silver Nitrate. Appl. Environ. Microbiol., 69, 4278-4281.

Nakamura, T., Ishihara, M., Ohana, T., Tanaka, A., and Koga, Y. (2004) Sidewall modification of single-walled carbon nanotubes using photolysis of perfluoroazooctane. Diamond Relat. Mater., 13, 19711974.
Nonami, T., Hase, H., and Funakoshi, K. (2003) ApatieCoated Titanium Dioxide Photocatalyst. Materials Science Forum, 439, 337-343.

Park, K.A., Choi, Y.S., Lee, Y.H., and Kim, C. (2003) Atomic and electronic structures of fluorinated single-walled carbon nanotubes. Phys. Rev. B, 68, 045429.

Ramanathan, T., Fisher, F.T., Ruoff, R.S., and Brinson, L.C. (2005) Amino-Functionalized Carbon Nanotubes for Binding to Polymers and Biological Systems. Chem. Mater., 17, 1290-1295.

Saito, R., Dresselhaus, M.S., and Dresselhaus, G. (1998) Physical Properties of Carbon Nanotubes. Imperial College Press, London.

Sondi, I., and Salopek-Sondi, B. (2004) Silver nanoparticles as antimicrobial agent: a case study on E. coli as a model for Gram-negative bacteria. J. Colloid Interface Sci., 275, 177-182.

Stevens, J.L., Huang, A.Y., Peng, L.W., Chiang, L.W., Khabashesku, V.N., and Margrave, J.L. (2003) Sidewall Amino-Functionalization of Single-Walled Carbon Nanotubes through Fluorination and Subsequent Reactions with Terminal Diamines. Nano Lett., 3, 331336. 